Originalveröffentlichung in: Annette Imhausen, Tanja Pommerening (Hg.), Writings of early scholars in the ancient Near East, Egypt, Rome, and Greece. Translating ancient scientific texts (Beiträge zur Altertumskunde 286), Berlin ; New York 2010, S. 69-91

\title{
Präzision in der Prognose oder: \\ Divination als Wissenschaft
}

\section{Joachim Friedrich Quack (Ruprecht-Karls-Universität Heidelberg)}

Unter dem Stichwort, Wissenschaftssprache` über Divination sprechen zu wollen, erscheint zunächst als erstaunliche Dreistigkeit. Wie kann man nur auf die Idee kommen, abergläubische Verfahren derart einzustufen, denen allenfalls eine Etikettierung als ,Pseudowissenschaft ${ }^{6}$ gerecht werden würde? Heutzutage versucht man zwar immer noch recht intensiv, die Zukunft vorherzusagen, verwendet dafür allerdings ein methodisches Rüstzeug insbesondere der Sozial- und Wirtschaftswissenschaften, von dem wir durchaus beanspruchen, dass es wissenschaftlich sei (und auch alle unvorhergesagten Katastrophen sowie fast regelmäßig merkliche Abweichungen von der Realität ändern an dieser Einstufung im allgemeinen Bewusstsein nichts).

Der Grund für meinen Zugang liegt natürlich in der Betrachtungsweise der Ägypter selbst. Ich beabsichtige im Folgenden zu zeigen, dass in der emischen Perspektive divinatorische Traktate im Alten Ägypten als wissenschaftliche Texte zu gelten haben. Allerdings sollte ich einschränkend gleich die wesentliche Frage stellen, in welchem Sinne man für Alte Kulturen überhaupt von Wissenschaft sprechen kann. Gibt es tatsächlich ein eigenkulturelles Konzept, das unserem Begriff, Wissenschaft ${ }^{\star}$ in irgendeiner Form entspräche? Und wie sähe dies aus? Würde man etwa diejenigen Kompositionen auswählen, welche explizit den Begriff, Wissen' in der Überschrift tragen, ${ }^{1}$ so fiele das Ergebnis schockierend aus. Zwar würde der Traktat über die Körpergefäße aus dem Papyrus Ebers darunter fallen (pEbers 99, 1; Nr. 854), aber vorrangig würde religiöses Wissen etwa im Stil des Amduats, der Sprüche für das Kennen der beiligen Seelen oder der Gaumonographien vertreten sein. Man sollte somit für Ägypten den Begriff von, Wissen-

1 Vgl. die Auflistung in SCHOTT (1990): 279-285. 
schaft ${ }^{6}$ von vornherein ganz anders angehen als wir es in unserer heutigen Wissenskultur gewohnt sind. Als Arbeitsdefinition werde ich hier anwenden, dass es systematisch aufgebaute Kompositionen seien, welche von einer intellektuellen Elite genutzt wurden, die davon ausging, dass es sich um gute und abgesicherte Verfahren handelte, um Wissen nicht-trivialer Art zu gewinnen oder gewünschte Wirkungen jenseits simpler Alltagsverfahren zu erzielen. In diesem Sinne möchte ich die divinatorischen Texte als Bestandteil der Wissenschaft nachweisen. Dargelegt werden soll dies anhand der Fundzusammenhänge der Handschriften, ihrer äußeren Gestaltung, der inneren Textstruktur sowie der präzisen Fachsprache.

Um eine einigermaßen homogene Gruppe für die Untersuchung zu erhalten, werde ich mich auf Traktate in demotischer Schrift konzentrieren. Einerseits ist die Basis hier besser, da es mehr substantielle Texte gibt, die entweder veröffentlicht oder mir sonst zugänglich sind als in älteren Perioden. Andererseits kann die Untersuchung der Fachsprache sinnvollerweise nur an einem linguistisch homogenen Korpus unternommen werden; ein Kontrollblick auf mögliche längerfristige Kontinuitäten kann erst im Anschluss daran erfolgen. Die von mir genutzten Quellen stammen somit als Handschriften im Wesentlichen aus dem 1. und 2. Jh. n. Chr., auch wenn sie fallweise bzw. für Teilpartien auf ältere Vorlagen bis zum 7. Jh. v. Chr. zurückgehen könnten.

Hauptquelle für mich sind zum einen Traumbücher, ${ }^{2}$ zum anderen terrestrische Omina, wobei ich gerade für den letzteren Bereich, der in Publikationen wenig greifbar ist, derzeit einen sehr substantiellen unpublizierten Textvertreter bearbeite. ${ }^{3}$ Daneben werde ich auch astrologische Traktate heranziehen, soweit sie derzeit bereits publiziert vorliegen, insbesondere den pBerlin 8345. ${ }^{4}$

2 Die wichtigsten Publikationen hierzu sind VOLTEN (1942); TAIT (1977): 5660, Taf. 4; ZAUZICH (1980): 91-98, T. 7-8; QUACK/RYHOLT (in Vorbereitung). Vgl. die Übersetzung einiger Abschnitte in QUACK (2008): 359-362. Zu den späthieratischen Traumbüchern siehe QUACK (2010): 99-110 und Taf. 34 -37. Für die älteren Epochen vgl. SZPAKOWSKA (2003).

3 Vorbericht in QUACK (2006a): 175-179. Da die endgültige Platzierung der Fragmente noch nicht feststeht, zitiere ich aus diesem Text ohne Stellenangabe.

4 SpIEgelberg (1902): 28, Taf. 97; Bearbeitung HugHES (1983): 53-69; Übersetzung QUACK (2008): 368-370. 
Zunächst sei der Fundzusammenhang der betreffenden Handschriften angesprochen. Eine erste substantielle Gruppe stammt aus Tebtynis am Südrand des Fayums. Soweit man es bei der sehr fragmentierten Erhaltung und dem für viele Teile aus dem Kunsthandel kommenden Bestand noch sagen kann, stammt die Hauptmasse dieses Materials aus zwei unterirdischen Räumen in der Nähe des Tempelbezirks. ${ }^{5}$ Das erhaltene Textmaterial dieses Fundkomplexes umfasst vorrangig im weitesten Sinne literarische und religiöse Texte. ${ }^{6}$ Daneben gibt es mathematische, ${ }^{7}$ medizinische, ${ }^{8}$ und astronomische Texte, ${ }^{9}$ deren wissenschaftliche Natur auch nach heutigen Kriterien unproblematisch ist. Nutzer dieser Textgruppe war eine ägyptischsprachige lokale Elite, die eng mit dem Betrieb am Tempel des Sobek verbunden war.

Ganz ähnlich sieht der Befund für den anderen wesentlichen Fundort, nämlich Soknopaiou Nesos am Nordrand des Fayums aus. ${ }^{10}$ Auch dort dürfte es sich um Texte handeln, die von Priestern genutzt wurden, und auch dort sind eindeutig wissenschaftliche Texte, insbesondere medizinische ${ }^{11}$ und astronomische, ${ }^{12}$ sicher nachgewiesen.

Wir können somit festhalten, dass die Traktate, um die es mir im Folgenden geht, in der indigenen intellektuellen Elite zirkulierten und gemeinsam mit Texten genutzt wurden, deren wissenschaftlicher Charakter auch nach heutigen Maßstäben unbezweifelt ist.

Eine weitere Frage kann im Zusammenhang mit der Zirkulation aufkommen, nämlich diejenige nach der Verbindlichkeit der Texte, also konkret der Kanonbildung, die ja für die mesopotamischen divinatorischen Texte ein gut bekanntes Phänomen darstellt. ${ }^{13}$ Das verfügbare Material zur Überprüfung ist im ägyptischen Bereich bislang allerdings

5 Vgl. hierzu etwa BEGG (1998): 185-210.

6 Vgl. bes. RYHOLT (2005): 141-170.

7 Ediert in PARKER (1972): 2, 5, 73-77, Taf. 25.

8 Weitgehend noch unpubliziert, siehe den Überblick von ZaUZICH (1991): 111, bes. 8; für ein bereits teilweise publiziertes botanisches Werk siehe TAIT (1977): 67-71, Taf. 5; TAIT (1991a): 47-92; für ein weiteres Fragment RYHOLT (2005).

9 Einige ediert bei Neugebauer/PARKER (1969): 240-243 u. 250-255.

10 Vgl. dazu mehrere Beiträge in: LIPPERT/SCHENTULEIT (2005).

11 Geplante neue Gesamtedition HofFmanN (in Vorbereitung).

12 So pWien D 4876, ediert von Neugebauer/PARKer (1969): 243-250; ferner HOFFMANN (1995): 22-26.

13 Vgl. HEEßEL (2008). 
gering, da kaum je inhaltlich gleichartige Passagen in mehreren Handschriften vorhanden sind. Im Detail ergibt sich dabei folgender Befund: Bei den Tieromina scheint es zumindest Spielraum für unterschiedliche Traditionen zu geben. Jedenfalls gibt es in Heidelberg einen Papyrus mit Omina, die auf dem Verhalten von Spitzmäusen beruhen, ${ }^{14}$ der sich völlig von den Spitzmausomina im großen Papyrus über die terrestrischen Omina unterscheidet. Dagegen ist es mir inzwischen gelungen, bei den Traumbüchern eine Passage nachzuweisen, die quasi exakt gleich in zwei verschiedenen Handschriften vorliegt, von denen eine aus Tebtynis stammt, die andere aus Soknopaiou Nesos. ${ }^{15}$ Damit gibt es also einen ersten Hinweis darauf, dass die Traumdeutung einen kanonischen und verbindlichen Textzustand erreicht haben könnte. Möglicherweise noch weit üppiger bezeugt ist ein unpubliziertes Losbuch, von dem ich wenigstens fünf verschiedene Handschriften kenne. ${ }^{16}$ Allerdings gibt es dabei nach derzeitigem Kenntnisstand nirgends sich überlappende Abschnitte; die Zuweisung zu einer einzigen Komposition beruht lediglich auf dem gleichen formalen Aufbau der Texte.

Nunmehr kann es an die innere Struktur der Traktate gehen. Wenigstens für die Traumbücher ist schon lange bekannt, dass gerade die spätzeitlichen sich durch eine systematische Anordnung nach Themen auszeichnen. ${ }^{17}$ Sämtliche für eine Überprüfung ausreichend gut erhaltenen demotischen Handschriften dieser Art haben auch explizite Überschriften zur Einleitung von Sektionen. So gibt es „Arten von Dingen, ${ }^{18}$ von denen ein Mensch träumt, wenn [eine Frau] davon träumt.“ (pCarlsberg 13, b, 2,14) oder: „Die Arten von Bier, von denen ein Mann träumt“ (pCarlsberg 14 vs., a 1); „die Arten von Dingen, wovon ein Mann träumt, dass man sie ihm sagt" (pCarlsberg 14 vs., c,2,2); „die Arten des Schwimmens, [wovon ein Mann träumt]"

14 Publiziert von SpIEgelberg (1925): 9-11.

15 Details in QUACK/RYHOLT (in Vorbereitung).

16 Text kurz erwähnt bei QUACK (2006a): 182-184.

17 VOLTEN (1942): 6-8, 15-16 u. 66; m. E. zu skeptisch TAIT (1977): 59. Vgl. QUACK (2010): 108-110.

18 So die Schreibung der Handschrift, eventuell ist $n k t$ „Ding“ Verschreibung für $n k$,Geschlechtsverkehr“. 
(pCarlsberg 14 vs., f, 16); ${ }^{19}$ „Die Arten des Säugens“ (pJena I 1209,1); „andere Geräte, [von denen] ein Mann träumt“ (pBerlin 15683, 2). Das bislang allerdings sehr dürftige späthieratische Material könnte darauf hindeuten, dass eine derartige Systematisierung und Überschriften sich erst in der Spätzeit herausbilden.

Auch die Tieromina zeigen eine derartige systematische Anordnung. Standard für sie ist die Einleitung n3 shn.w $n$ n3 „die Vorzeichen der" mit anschließender Bezeichnung der konkret behandelten Spezies. Vergleichbare Erscheinungen finden sich in den astrologischen Traktaten, in denen z. B. n3 shn.w $n$, die Vorzeichen des" mit anschließender Nennung des jeweiligen Planeten vorkommt. ${ }^{20}$

Wo eine innere Systematisierung des Materials innerhalb eines Abschnittes sich anbietet, wird sie auch konkret durchgeführt. So gibt es bei den Tieromina ausführliche Sektionen darüber, was es bedeutet, wenn kleinere Tiere, z.B. Mäuse, mit bestimmten Körperteilen eines Mannes in Kontakt kommen. Hier wird die Abfolge der Körperteile vom Kopf zu den Füßen gleichartig wie in medizinischen Texten und Heilritualen durchgeführt. Astrologische Traktate gehen jeweils systematisch in standardisierter Abfolge entweder die Position eines Planeten in jedem Haus bzw. Tierkreiszeichen durch oder für jedes Haus sämtliche Planeten.

In ihren Formulierungen sind die Traumbücher und terrestrischen Omina normalerweise kasuistisch, also im Stile von Bedingungsgefügen. Es gibt also Formulierungen wie „Wenn seine Füße an seinem Kopf sind: Er wird ein gutes hohes Alter erreichen." (pCarlsberg 13, b, 2, 7). Als Beispiel aus den terrestrischen Omina genannt sei etwa: „Wenn eine Maus in einem Haus Kot macht, ist es gut für den Hausherrn in selbigem Monat. "Bei repetitiven Passagen kann das erste Element verkürzt werden, indem man den Konditionalis selbst weglässt und lediglich noch das Omen anzeigende Objekt bzw. den Körperteil nennt.

Für die Protasis werden zwei verschiedene sprachliche Realisierungen gewählt. Die erste ist der normale Konditionalis des Demotischen, der mit Substantiven in-n3.w A sçm, mit Suffixen ỉw=f sçm

19 Schlecht erhaltene Abschnittsüberschriften auch pCarlsberg 13, d, 8; pCarlsberg 14 vs. a $16 ; c, 2,12 \mathrm{f}$.

20 So im pBerlin 8345. 
lautet. ${ }^{21}$ Daneben findet sich auch die Verwendung des zweiten Tempus $i: i r i=f s c ̧ m$, bei dem Substantive und Suffixe gleich flektiert werden. Der Gebrauch der zweiten Tempora im Demotischen für Bedingungssätze ist schon mehrfach diskutiert worden, wobei diese Verwendung zuletzt auf die ,Wechselsätze ${ }^{\varsigma}$ des Mittelägyptischen zurückgeführt wurde. ${ }^{22}$ Die semantische Abgrenzung vom normalen Konditionalis ist bisher nicht gelungen. Gerade das Nebeneinander in den divinatorischen Traktaten macht die Sache nicht leichter. Es fällt schwer, in den Seite an Seite verwendeten Konstruktionen inhaltliche Unterschiede festzustellen. Möglicherweise handelt es sich um eine entweder diachron signifikante Unterscheidung ${ }^{23}$ oder eine eher stilistisch bedingte, bei der auch mit der Verwendung inhomogener Quellen gerechnet werden muss.

Sofern die Protasis nicht aus einem einzigen Verb mit Satelliten besteht, sondern eine Abfolge mehrerer kombinierter Beobachtungen vorliegt, wird nach dem satzeröffnenden Konditionalis die Protasis durch den Konjunktiv, gegebenenfalls auch eine Kette von Konjunktiven fortgeführt. Dabei liegt eine logische Und-Verknüpfung vor, d.h. das Eintreten sämtlicher Elemente der Protasis ist Voraussetzung für das Eintreten der Apodosis. Sofern bei gleichbleibenden Teilelementen der Protasis einzelne Details abweichen, werden die neuen Elemente mit $i w=f h p r$ und Umstandssatz „Falls aber ...“ eingeführt.

Die bei weitem häufigste Form der Apodosis ist das Futur III, mit

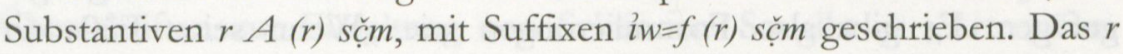
des Futurs bleibt oft ungeschrieben, ist nach Maßgabe der Pleneschreibungen jedoch überall anzusetzen. ${ }^{24}$ Sofern die Apodosis aus mehreren Sätzen besteht, wird jeder einzelne mit Futur III eingeleitet; der Gebrauch des Konjunktivs ist hier nicht belegt. Negierte Apodosis ist selten; sie wird mit dem negierten Futur III $b n-i ̂ w=f s c ̧ m m$ konstruiert. Bei Eigenschaftsverben wird, sofern man abstrakt „es“ formuliert, statt des Futurs die spezielle Form mit $n$ 3-Präfix gebraucht, so etwa $n$ 3- $n f r=s$

21 Als Reliktform auch in-i $(w)=f$ sç $m$ und $i n-n 3 . w-i ̂ w=f s c ̧$

22 SIMPSON (1996): 172 mit Verweisen auf die ältere Literatur; siehe QUACK (2006c): 259-260.

23 Die Belege in den Texten liefern dafür allerdings keinerlei Hinweis.

24 Zur Defektivschreibung im Futur III siehe JOHNSON (1976): 155-157; der Versuch von HuGHES (1983): 58 (zu I/2), Formen ohne $r$ weitestmöglich als Präsens zu verstehen, dürfte weniger empfehlenswert sein. 
„es ist gut". Formuliert man dagegen konkret auf eine Person, wird auch bei diesen Verben das Futur verwendet, also $i w=f r n f r$. $t$,,er wird es gut haben.". Selten ist der Aorist hr sç̌m $=f$ belegt.

Spezielle sprachliche Formulierungen finden sich bei ungünstigen Omina, deren Wirkung abgewendet werden soll. Relativ selten ist der einfache Imperativ in der Form hrh $r . r=f$, achte auf ihn“. Sehr viel häufiger ist der Optativ $m y$ sç̣m= $f$ „möge er hören“, der stets eine Aufforderung beinhaltet, durch bestimmte Handlungen (Flehen zu den Göttern oder Schutzmagie) schlechte Vorzeichen abzuwenden. Negatives Gegenstück zum Optativ ist der negierte kausative Imperativ, der immer in der Verbindung $m$-iri č či.t šm $=f$ auftritt. ${ }^{25}$ Er bedeutet zwar etymologisch gesehen ursprünglich „Lass ihn nicht gehen“, ist nach

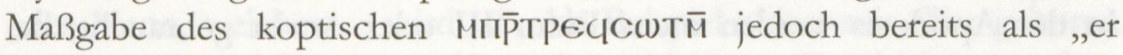
soll/möge nicht gehen " zu verstehen. ${ }^{26}$ Neben den weitaus dominierenden Verbalsätzen finden sich in der Apodosis selten Substantivalsätze, die teilweise allein stehen, meist jedoch mit einer verbalen Apodosis verbunden sind.

Das sprachliche Muster lädt zum Vergleich mit ähnlich konstruierten Sätzen in anderen Textgattungen ein. ${ }^{27}$ Die eine sind Weisheitslehren, in denen sich Bedingungssätze vor allem in den mehr kasuistischen älteren Kompositionen als Einleitung vieler oder sogar aller Maximen finden. ${ }^{28}$ Gerade in den mit den hier behandelten Traktaten etwa zeitgleichen demotischen Weisheitslehren finden sich jedoch Bedingungssätze nur sporadisch; zumindest in den bisher publizierten Texten. ${ }^{29}$ Dies hängt damit zusammen, dass die demotischen Lebenslehren eben nicht mehr kasuistische Maximen bieten, sondern entweder lose Einzelsätze mit allenfalls assoziativer Verbindung oder

25 Für Belege in Omentexten siehe VOLTEN (1942): $93 \mathrm{zu} \mathrm{b,} \mathrm{x}+3$.

26 Vgl. Till (1966): § 346.

27 Vgl. methodisch ähnlich RITTER, in diesem Band.

28 Durchgängig findet sich diese Formulierung in der Lebre des Ptabhotep und der Lebre für Kagemni, ferner im vorderen Bereich der Lebre für Merikare sowie im hinteren Bereich der Berufssatire.

29 Ein Text vermutlich aus Tebtynis, der früher in Oxford mit der provisorischen Nummer pOxy. 79/103 und 104 aufbewahrt wurde und sich heute in Berkeley befindet, beginnt jedoch zumindest in den meisten Fragmenten jeden Abschnitt (etwa alle drei Zeilen) mit einem graphisch nach rechts ausgerückten Konditionalis; siehe QUACK (im Druck). 
das Umreißen eines bestimmten Themas mit ebenfalls selbständigen Sätzen. ${ }^{30}$

Interessanter ist der Vergleich mit einer anderen Textgattung, nämlich den Gesetzestexten. Die inzwischen bekannten demotischen Gesetzestexte ${ }^{31}$ zeigen zwei Arten der Formulierung. Besonders in der Zivilprozeßordnung, aber auch im Codex Hermopolis (IX, 3.4.8) ist der

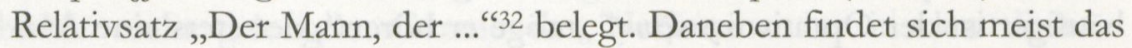
Bedingungsgefüge. Dabei beginnt die Protasis üblicherweise mit dem normalen Konditionalis, der durch Konjunktiv fortgeführt werden kann. Das zweite Tempus in konditionaler Verwendung ist selten belegt (Codex Hermopolis III, 1.14; VI, 14). Für modifizierende Abweichungen wird $i w=f h p r$ mit Umstandssatz „Falls er aber ..." verwendet. In der Apodosis werden meist Futur III oder Aorist gebraucht; bei negierter Formulierung ist ausschließlich der negierte Aorist $b w$-iri $i=f$ sç̌m bezeugt. ${ }^{33}$ Dabei wird, wenn die Apodosis aus mehreren Sätzen besteht, der Aorist mit Konjunktiv fortgeführt, während beim Futur III mehrere gleichartige Formen hintereinander gebraucht werden (Codex Hermopolis I, 11f.; II, 6.8; II, 13.19; IV, 10.19; V, 5f.19.23f.26f.; VI, 10.11; VII, 2; VIII, 17; IX, 19; mit zusätzlichem gr VII, 8.13).

30 Vgl. QUACK (2009): 111-112.

31 Wichtige Editionen SPIEgELberg (1929); SETHE/SPIEGELbERG (1929); SEIDL (1963); KAPLONY-HECKEL (1986); MATTHA (1975); BRESCIANI (1981): 201-215; TAIT (1991b): 93-99; CHAUVEAU (1991): 103-127; LIPPERT (2003): 91-135; LIPPERT (2004a); LIPPERT (2004b): 389-405. Siehe den Überblick in LIPPERT (2008). Für den im Folgenden hauptsächlich herangezogenen Codex Hermopolis habe ich die handliche Edition von DONKER VAN HEEL (1990) zugrunde gelegt.

$32 \mathrm{Zu}$ diesem Grundtyp der Gesetzesformulierung siehe YARON (1988): 105f., 109f. $\mathrm{Zu}$ ägyptischen Belegen dieser Formulierungsweise siehe LORTON (1977): 53-59, dem ich allerdings nicht darin folgen möchte, diesen Aussagetyp für die im älteren Ägyptisch allein übliche, von Vorderasien unabhängige Bildung zu halten. Einerseits existiert sie auch in Vorderasien, andererseits stammen die bisher bekannten Belege des älteren Ägyptisch nicht aus Gesetzeskodizes, sondern sind Einzelfestlegungen von Dekreten und verwandten Texten. Ähnlich wie die Dekrete formulieren auch die Drohformeln, siehe MORSCHAUSER (1991): 6-20.

33 Hier zeigt sich eine interessante Parallele zum astrologischen Text pBerlin 8345, der positive Prognosen meist als Futur III, negierte jedoch immer als negierten Aorist formuliert; vgl. HuGHES (1983): 54. 
Gelegentlich findet sich als Apodosis auch eine Cleft-Sentence (Codex Hermopolis VI, 1; VII, 5; VIII, 30; IX, 30).

Insgesamt gesehen gibt es kleine Unterschiede zwischen den kasuistischen Omentexten und den Gesetzestexten. So zeigen letztere eine häufigere Verwendung des Aoristes; zudem sind die Protasen oft erheblich komplizierter verschachtelt. In der äußeren Anlage der Komposition kann man aber dennoch ziemliche Ähnlichkeiten feststellen. Diese Berührungspunkte dürften auf einen vergleichbar ernsthaften Umgang mit dem Material sowie eine ähnliche geistige Fähigkeit zur Durchdringung des Stoffes seitens der Autoren hindeuten. ${ }^{34}$

Auffällig abweichend sind die astrologischen Traktate gebildet. In ihnen dominiert gerade die nichtkasuistische Stilisierung in der Art von „der Mann, welcher ..., wird ...“. Als Beispiel zitiert sei „Wer geboren wurde, während Venus im Haus des schlechten Geschicks war, dem wird viel übler Leumund zuteil werden, während er jung ist. Er wird mit einer Frau wirr gehen. Wenn er alt geworden ist, wird er aufhören, und sein Herz wird mit einer Ehefrau glücklich sein." (pBerlin 8345, 2, $1-4)$. Dies ist also gerade das Schema, das man aus manchen Gesetzestexten kennt - vielleicht nicht unpassend, denn gerade die astrologischen Traktate sind diejenigen, welche am meisten mit einem festen gesetzesmäßigen Eintreffen der Prognosen rechnen und an sich keinen Raum dafür lassen, durch Gebete das Schicksal noch zu modifizieren. ${ }^{35}$

34 Im alten Orient, wo man die ähnliche Textorganisation von Gesetzestexten und Omenliteratur schon lange gesehen hat, wurde daraus gelegentlich auf den mehr literarisch-künstlichen als den normativ verbindlichen Charakter der Gesetzestexte geschlossen, siehe etwa Kraus (1960): 283-296. Nach meiner Ansicht ist diese Argumentation nicht stichhaltig und dürfte bestenfalls auf den Kopf zu stellen sein: Wenn die Omentexte ernst genug genommen wurden, dass der assyrische König sich ständig über Vorzeichen auf dem Laufenden hielt und im Zweifelsfall aufwendige Rituale zur Abwehr des angekündigten Unheils durchführen ließ (siehe MAUL (1994): 17-36), wird man auch mit der realen Bedeutung der Gesetzestexte rechnen müssen, sofern man eine Art Junktim zwischen den beiden Textgattungen als sinnvoll erachtet. Für tatsächliche Anwendung sowohl der Omensammlungen wie der Gesetzestexte plädiert auch WESTBROOK (1985): 247-264; FINCKE (20062007): 139-147 betont ebenfalls die Verbindung zwischen diesen Textsorten. 
Als Ergebnis dieser textlinguistischen Untersuchung kann man Folgendes festhalten: Mit ihrer sorgfältigen Anordnung des Materials und dem klaren sprachlichen Aufbau erweisen sich die divinatorischen Traktate als Produkt eines Geistes, dem man nur gerecht wird, wenn man ihn als wissenschaftlich bezeichnet. Die Formulierungen sind demotischen Gesetzestexten ähnlich, andere ägyptische Omentexte zeigen auch einen sprachlichen Aufbau, der sich vergleichbar in medizinischen Texten wiederfindet, die als Spitzenleistungen ägyptischer Wissenschaftlichkeit angesehen werden. ${ }^{36}$ Man sollte die Texte also als Wissenschaft der damaligen Zeit betrachten, statt in ihnen reinen Aberglauben $\mathrm{zu}$ sehen, ${ }^{37}$ wie ja auch für die mesopotamische Divination der Status als Wissenschaft proklamiert wurde. ${ }^{38}$ Die nächste Aufgabe wird sein, durch Analyse des Inhalts festzustellen, nach welchen Prinzipien diese Wissenschaft operiert.

Besonders für die mesopotamische Divination ist schon intensiv diskutiert worden, inwieweit sie auf realer empirischer Beobachtung beruht. ${ }^{39}$ Generelle Tendenz der neueren Forschung ist dabei, weniger

36 Vgl. etwa VERNUS (1981).

37 Gegen eine Bewertung als Aberglaube wendet sich im Hinblick auf die Tagewählkalender auch LEITZ (1994): 485f. Das hier zunächst auf der formalen Ebene gewonnene Ergebnis deckt sich weitgehend mit der von VOLTEN (1942): 40-44 betonten engen Verbindung von Magie, Traumdeutung und Wissenschaft und seiner Auffassung der Traumdeutung als ägyptischer Wissenschaft.

38 JEYES (1991-1992): 23-41; ROCHBERG (1999): 559-569; BROWN (2000): 227-229; SOMMERFELD (2000): 201-219; MAUL (2003-2005): 45-88.

39 Fossey (1921-1922): 1-18 arbeitet grundlegend Symmetrie- und Sympathiebeziehungen heraus. JASTROW jr. (1914): 2f. und passim nennt Ideenassoziation und Beobachtung von tatsächlichen Ereignissen als Grundelemente der divinatorischen Deutung. LABAT (1951): XXXI-XXXIV gibt zwar Indizien an, die für spekulative Ausarbeitung sprechen könnten, betont jedoch vorrangig die tatsächliche Beobachtung. PETTINATO (1966): 218-222 spricht vor allem die Assoziation an. LEICHTY (1970): 20 vermutet, dass ursprünglich tatsächlich beobachtete Vorzeichen später theoretisch expandiert wurden, wobei auch einige unmögliche Fälle aufgenommen wurden. STARR (1983): 3 u. 7-9 geht von einer ursprünglich empirischen Grundlage der Divination aus, wobei die Systematisierung und Anordnung des Materials in Serien zum Aufhören der Einzelbeobachtung geführt hätte. KOCH-WESTENHOLZ (1995): 13-19 nimmt dagegen an, dass weniger Empirie zugrunde liegt, sondern eine Theorie von der inneren Verbundenheit des Universums. 
Empirie anzusetzen und mehr globale Vorstellungen über die innere Verbundenheit der Welt. Entsprechendes dürfte auch für das Alte Ägypten gelten, wo diese Diskussion bislang kaum geführt worden ist.

Als Hauptmittel der Divination kann man zwei Verfahren erkennen, mit denen aus den Vorzeichen selbst eine Deutung generiert wird. ${ }^{40}$ Einerseits ist dies das Wortspiel, andererseits eine symbolischassoziative Verbindung. Das Wortspiel ist in älteren ägyptischen divinatorischen Texten wie dem Traumbuch des pChester Beatty III, einer ramessidischen Handschrift (ca. 1300 v. Chr.), die mutmaßlich auf einen noch älteren Prototyp zurückgeht, ${ }^{41}$ relativ häufig. ${ }^{42}$ In den demotischen divinatorischen Traktaten findet es sich dagegen nur noch in einer Sektion, nämlich in einem Abschnitt im Traumbuch des pCarlsberg 13, in dem es um Träume von Zahlen geht. Praktisch alle Vorhersagen beruhen auf Lautähnlichkeit. ${ }^{43}$ Für die Zahlen dürfte es auch wenig andere Möglichkeiten der Ausdeutung geben.

Dagegen dominiert in allen anderen Bereichen die symbolischallegorische Ausdeutung. Sie ist auf jeden Fall einen genaueren Blick wert, weil sich darin jenseits der generellen Einstufung auch zeigt, wie diese Denkweise konkret funktioniert. ${ }^{44}$ Einige Fälle sollen hier beispielhaft präsentiert werden.

In einem noch unpublizierten Traumbuch (pCarlsberg 649 vs.) gibt es mehrere Träume von Fischen. „Sonstige“ Fischsorten zeigen dabei an, dass dem Träumer Speise gebracht werden wird, was naheliegt, da

Verschiedene assoziative Methoden speziell der Traumdeutung nennt ZGOLL (2006): 457-462.

40 Vgl. in diesem Sinne LeITZ (2000): 221-246, bes. 226-234.

41 Vgl. QuACK (1997): 277-287.

42 Vgl. etwa NOEGEL (2007): 89-106, auch wenn ich sein Postulat vorderasiatischer Einflüsse mit Skepsis ansehe, da das Wortspiel an sich in Ägypten schon seit dem Alten Reich ein gut belegtes Verfahren der Sinnstiftung darstellt; siehe etwa SANDER-HANSEN (1948): 1-22; MORENZ (1958): 23-32; wieder abgedruckt (1975): 328-342; LOPRIENO (2000): 3-20.

43 Vgl. die Detailnachweise bei QUACK (2008): $359 \mathrm{f}$.

44 Die Untersuchung des Systems von Assoziationen, welche den divinatorischen Prognosen zugrunde liegen, wurde schon von ZAUZICH (1980): 91f. als wichtig herausgestrichen; bereits in GARDINER (1935): 9-23; VOLTEN (1942): 50-66; SZPAKOWSKA (2003): 77-114 finden sich scharfsinnige Einsichten. 
Fisch ein relativ wichtiges Nahrungsmittel in Ägypten ist. ${ }^{45}$ Von einem Fisch zu träumen, der als „Schlange des Flusses“ bezeichnet wird, soll dem Träumer anzeigen, dass er sich mit Speise anfüllen wird. Unabhängig davon, ob der betreffende Fisch ein Aal, ein Flösselhecht oder eine andere Art darstellt, darf man annehmen, dass es sich um einen Raubfisch handelt, dessen Verhalten auf den Träumer übertragen wird. Der Traum von frischem Fisch zeigt an, dass einem Geheimnisse offenbart werden. Dies ist insofern verständlich, als frischer Fisch zur weiteren Verarbeitung aufgeschnitten werden muss, man also die verborgenen Innereien zu sehen bekommt. Gesalzener Fisch ist ein Hinweis darauf, dass man Vorräte vom Speicher nehmen wird natürlich deshalb, weil gesalzener Fisch ein länger haltbares Speicherprodukt ist. Dagegen erfährt man vom gekochten Fisch, man werde rasch Nahrung erhalten - dies ist eben ein Produkt zum sofortigen Verzehr.

Als zweiten Fall möchte ich einige Beispiele aus dem Bereich der terrestrischen Omina wählen. Diese sind im Allgemeinen schwieriger $\mathrm{zu}$ analysieren, da man mehr unterschiedliche Faktoren einbeziehen muss, nicht nur die Tierart an sich, sondern auch das konkrete Verhalten bzw. den Kontakt zu einem bestimmten Körperteil des Menschen. Dabei scheint in der Symbolik der Körperteil an sich die dominierende Rolle einzunehmen. Kontakt eines Tieres mit den Ohren eines Menschen kann ein Anzeichen dafür sein, dass man Botschaften hören wird. Die Lippen werden mit Verleumdung bzw. Essen zusammengebracht; d.h. also, dass Sprechakte bzw. Nahrungsaufnahme als für die Lippen typische Aktionen angesehen werden. Die Schultern werden gerne mit dem Tragen bzw. konkret mit dem Beenden von Lasten oder Mühen, die verschiedenen Bereiche von Armen und Händen mit dem Besitz und Erwerbsleben verbunden, daneben auch mit Gaben sowie Leitung (,an der Hand nehmen“). Der Bezug zum Erwerbsleben dürfte sich klären, wenn man bedenkt, dass im Ägyptischen iri $m h p s \check{s}=f$ „mit seinem starken Arm handeln“ u. ä. geläufige Ausdrucksformeln sind, die den Erwerb von Besitz durch eigene

45 Vgl. GAMER-WALLERT (1970); VAN ElSBERGEN (1997); in Deir el-Medineh, wo man am ehesten die Situation ,durchschnittlicher ${ }^{\star}$ Ägypter greifen kann, war Fisch als Eiweißlieferant wichtiger als Fleisch, siehe VALBELLE (1985): $272-274$. 
Arbeit bezeichnen. ${ }^{46}$ Das Herz wird im einzig erhaltenen Beleg in recht offensichtlicher Weise mit Herzensfreude zusammengebracht, die weibliche Brust zum einen als erogener Bereich mit Liebe verbunden, zum anderen mit dem Stillen von Kindern. Weniger spezifisch scheinen die meisten Prognosen für die unteren Körperpartien sich festlegen zu lassen: Geschäftsleben, Besitz und Versorgung stechen besonders hervor. Allerdings könnte die Logik sich daraus ergeben, dass solche Unternehmungen oft mit Geschäftsreisen assoziiert sind, welche die Beine involvieren.

Jenseits des Spezialfalles der Körperkontakte gibt es eine Reihe von Fällen, in denen der inhärente symbolische Charakter der vom Omenanzeiger vollzogenen Handlungen wichtiger erscheint als der Omenanzeiger an sich. Einige Belege dürften relativ klar sein: Der Anblick sterbender Tiere wird gerne mit dem Überwinden von Feinden zusammengebracht. ${ }^{47}$ Aus der Perspektive der Ägypter heraus, die Tierjagd oder Tieropferung mir Feindvernichtung parallelisieren, ${ }^{48}$ ist diese Deutung sehr einsichtig.

Tiere, die vor den Augen des Beobachters Futter verzehren, künden oft gute Versorgung mit Speise an, aber auch drohenden Verlust wegen Völlerei. In beiden Fällen dürfte das direkte Essen an sich der Deutung zugrunde liegen, die Details dagegen sind artabhängig. Wenn die Tiere dagegen Speise nicht direkt fressen, sondern davontragen, wird mit Verlust, Mangel und schlechter Versorgung gerechnet. Zugrunde gelegt wird dabei wohl die Vorstellung, dass es nötig ist, Vorräte zu horten, weil die Versorgung schlecht wird.

Der Kontakt eines Tieres mit einer menstruierenden Frau ist ein sicheres Anzeichen baldiger Schwangerschaft. Hier dürfte das Konzept zugrunde liegen, dass gerade in einer kritischen Phase ein ,Impuls' von außen hinzukommt. Ein recht plakativer Symbolismus ist es, wenn vom Herauskommen einer Maus zwischen den Beinen einer Frau auf ein Kind geschlossen wird. Schließlich droht, falls das Tier nach kurzem Kontakt sofort wegspringt, eine Fehlgeburt. Dabei dürfte das oben dargelegte Konzept der Schwängerung erweitert sein, indem die

\section{Vgl. etwa JANSSEN (1946): $75 f$.}

47 Vgl. hierzu in der älteren Traumdeutung pChester Beatty III rt. 3, 13; 4, 10. 16; ähnlich wird in pChester Beatty III rt. 5, 3 das Trinken von Blut als das Auslöschen von Feinden gedeutet.

48 HORNUNG (1967): 80; QUACK (2006b): 67-80. 
sofortige Entfernung des Tieres vom Körper als vorzeitige Entfernung des Embryos ausgelegt wird.

Auffällig ist ein weiterer Punkt, nämlich die Dimensionalität. Die Richtungsdimension rechts bzw. links tritt im Text einerseits in Verbindung mit Körperteilen auf, die in Kontakt mit dem Omenanzeiger treten, andererseits bei Handlungen von Tieren, welche diese zur Rechten oder Linken des Beobachters ausüben. Schließlich kann noch der Richtungswechsel von rechts nach links und umgekehrt relevant sein. Das Bild ist nicht einheitlich, aber tendenziell erscheint es doch so, dass die linke Seite deutlich häufiger positive Vorzeichen hervorbringt, die rechte dagegen vorwiegend negative.

Nun kann man prinzipiell annehmen, dass in der ägyptischen Kultur rechts die bevorzugte Seite war, wofür es auch genügend eigenkulturelle Belege gibt. ${ }^{49}$ Für die scheinbare Bevorzugung der linken Seite im Omentext lässt sich aber ein Erklärungsmodell aufstellen. Ausgangspunkt ist dabei die am mesopotamischen Omenmaterial gewonnene Erkenntnis, dass bei Missgeburtsvorzeichen eine Missbildung rechts schlecht, links gut ist, zwei Missbildungen dagegen rechts gut, links schlecht. ${ }^{50}$ Dabei wird offenbar gleichsam mathematisch minus mal minus als plus gewertet. ${ }^{51}$ Entsprechendes möchte ich für das ägyptische Material annehmen. Der Kontakt des Tieres mit einem Körperteil wurde tendenziell eher als schlecht bzw. gefährlich angesehen, wobei diese Bewertung in Verbindung mit der an sich schlechten linken Seite insgesamt zu einer tendenziell guten Endabrechnung führte.

Prinzipiell würde ich das Vordringen der symbolischen Ausdeutung auf Kosten des Wortspiels als geistesgeschichtliche Entwicklung im Sinne eines Fortschrittes bewerten. Sie ist eine deutlich komplexere intellektuelle Arbeit als die reine Reduktion auf den lautlichen Klang eines Wortes. Allerdings sollte man auch die Grenzen eines solchen Verfahrens herausstreichen, die eben dazu beigetragen haben, dass es heute nicht mehr wissenschaftlich akzeptabel ist. Assoziationen haben eine logische Verankerung im mentalen Verständnis der jeweiligen Kultur und ihres Symbolsystems. Sie sind jedoch weder

49 Siehe etwa MOREnZ (1957): 62-71; AUFRÈrE (1985): 31.

50 LEICHTY (1970): 7. Zu rechts und links in der mesopotamischen Divination siehe weiter FOSSEY (1921-1922): 4-11; STARR (1983): $15 f$.

51 Vgl. Koch-Westenholz (1995): $10 f$. 
kulturübergreifend generalisierbar, noch sind sie selbst innerhalb einer Kultur zwingend. Vielmehr könnte man sich selbst im Rahmen ägyptischer Weltsicht vielfach alternative Deutungen denken.

Schließlich möchte ich noch auf die Frage des Fachvokabulars zu sprechen kommen. An sich ist ein solches im Ägyptischen weniger leicht als in modernen Sprachen zu fassen, da hier z. B. die Option lateinischer und griechischer Termini fehlt. Dennoch kann man für ägyptische wissenschaftliche Texte gelegentlich sehen, wie Wörter mit einer engeren und präziseren Bedeutung verwendet werden, als an sich im Textspektrum möglich wäre. Hier sollen nur wenige Fälle besprochen werden, die mir besonders einschlägig scheinen. Eine Behandlung des gesamten Spektrums kann unmöglich erfolgen, es sei nur die Bemerkung erlaubt, dass die Phraseologie der divinatorischen Texte relativ fest ist und man deshalb mit genügender Texterfahrung auch schlechter erhaltene Stellen oft erfolgreich ergänzen kann.

Vor allem eine Wortverwendung ist mir aufgefallen: Das Substantiv $m t . t$, das an sich ein eher blasser genereller Begriff für „Sache, Angelegenheit" ist, dürfte in den divinatorischen Texten vielfach spezifisch ein wirtschaftliches Geschäft bezeichnen. Einige Beispiele sollen seine Verwendung illustrieren: $i w=f r \check{s} m n$ mt.t $r m n h w$ n. $i m=s$,er wird in ein Geschäft gehen, das keinen Profit hat"; iws=s 'r iri mt.t $r^{7}$ bn-iw hw n.im=s wrr $p 3 i$,sie wird ein Geschäft machen, an dem kein Profit ist; das bedeutet Verzögerung“; $r$ mt.t $r$ iri iw $n$ - $\breve{r r} . t=f$ „Ein Geschäft wird bei ihm eintreffen" bzw. ausführlicher $i w=f r$ či.t hpr nkt $n$ rmč ${ }^{3}$ r m t.t $r$ iri î $n$-črr.t $=f$, er wird reichen Besitz erwerben, ein Geschäft wird bei ihm eintreffen“; $i w=s$ r rwš $n$ mt.t $c_{\breve{s} 3 y} 3 n$ - iw $=w$ iri $n=s i w$, sie wird sich um viele Geschäfte bemühen und sie werden ihr nicht eintreffen“; n3.w$n f r=s n m t . t n b n t i-l i w=f r^{r}{ }^{r} m^{\gamma}[n$.im $m=w]$ „Es ist gut für jedes Geschäft, zu dem er geht“; $i w=f r \check{s} m w b 3$ mt.t $i w=s(r) c_{n} n$-čcr.t=f ,Er wird zu einem Geschäft gehen und es wird ihm gelingen"; $m$-iri či.t $3 s y$ „er soll nicht in ein Geschäft gehen, damit er keinen Verlust erleidet"; $i w=f$ hpr $i w$-iw $i w$ in- $n^{c} . k$. $t$ mt.t wrr $p^{3}[i]$ „Falls du zu einem Geschäft ausziehst, bedeutet das Verzögerung“".

Ein $m t . t$ ist also etwas, zu dem man sich aktiv hinbegibt, mit Verben des Gehens ausgedrückt, und zwar in den Dauerzeiten in- $\boldsymbol{n}^{c} . k . t$, sonst šm (das im Demotischen regulär nicht in Dauerzeiten konstruiert wird). Es ist, und dies ist mein weiteres Hauptargument, regulär mit Fragen finanziellen Gewinns oder Verlustes verbunden. Die dafür gebrauchten Ausdrücke sollte ich allerdings auch kurz erläutern: 
Ausdruck für wirtschaftlichen bzw. finanziellen Verlust ist ç̣.t. $3 s y$, was etymologisch zunächst nach „Verlust bewirken" klingt, somit so wirkt, als könnte es auch bedeuten, dass man jemand anderem Verlust zufügt. Angesichts von koptisch † oc€ „Verlust erleiden“ ist die konkrete Festlegung, wer wirklich schlechter dasteht, allerdings eindeutig. Daneben gibt es, von offensichtlicher Bedeutung, den Begriff hw „Profit", der in dieser Textgruppe normalerweise nur negiert vorkommt, somit bei seinem Auftreten zumindest das Ausbleiben von Gewinn signalisiert.

Gegensatz dazu ist somit der Gewinn, und dieser wird in den divinatorischen Traktaten regulär durch eine Wendung ausgedrückt, bei der eigentlich sogar die Lesung paläographisch zu diskutieren wäre. ${ }^{52}$ Dafür ist hier allerdings kaum das richtige Forum, so dass ich mich mit der Bemerkung begnüge, dass ich die Lesung iri î für sicher halte. Dabei handelt es sich um eine Formulierung mit einem Verbalnomen, die wörtlich „einen Eingang machen“ bedeutet. Diese Wendung ist in einer Reihe verschiedener Textsorten und Situationen belegt. (1) Eine Person macht das Eintreffen einer Sache, d.h. er quittiert ihren Eingang. ${ }^{53}$ Diese Verwendung findet sich in administrativem Zusammenhang. (2) Eine Personengruppe macht ein Eintreffen bei einem Ort, d.h. sie erreicht ihn. Dieser Gebrauch ist in demotischen Erzählungen belegt, so Amaz̧onen 2, 29f.; pKrall 8, 11.3. Ein konkretes Objekt macht ein Eintreffen bei einer Person bzw. annehmenden Stelle, d.h. es wird in Empfang genommen und verbucht und geht damit in den Besitz bzw. die Verfügung des Empfängers über. ${ }^{54}$ Auch dies ist in administrativen Texten normal; Vorläufer finden sich bereits im Neuägyptischen, wo iw „eingegangen“ in administrativen Ostraka vorkommt (z. B. HO XX 3, 3-5; XXIII 2, 11; L 1 rt. 2, 5 u. 7; LV 1 vs. 5 u. 6; LXI 4, 5). Schließlich gibt es das vorliegende Korpus. Hier haben wir zwar keinen konkreten Gegenstand als Subjekt, aber dennoch kann wohl kein Zweifel bestehen, dass ebenfalls der erfolgreiche Erwerb gemeint ist; in einem Fall ist die Wendung ja noch ganz deutlich mit „er wird reichen Besitz erwerben“ kombiniert.

52 Dies betrifft besonders die Ansetzung der Belege im Papyrus Krall als iri 3 , die zuletzt noch HofFMANN (1996): 199, Anm. 1017 u. S. 442 vertreten hat. Im Grundsätzlichen verweise ich auf RYHOLT (1999): 39 (zu 5, 19).

53 Vgl. hierzu HuGHES (1957): 58.

54 Belege zusammengestellt von SETHE/PARTSCH (1920): $110 \mathrm{f}$. 
Schließlich möchte ich noch ein Spezialproblem ansprechen. Möglicherweise in Zusammenhang mit dem Tod steht die Ankündigung $r$ p3 nb pr $r$ whm ${ }^{c} n h$,der Herr des Hauses wird das Leben wiederholen“ (Tieromina, ähnlich pCarlsberg 14, a, 10; pBerlin 8769, B $\mathrm{x}+2,2)$. Diese Prognose ist inhaltlich nicht unproblematisch, da whm $c_{n h}$ üblicherweise ein Epitheton von Verstorbenen ist (WB I: 341, 3-6), sich hier aber sicher auf einen Lebenden bezieht. Allerdings kann es auch auf erneuerte Statuen (WB I: 341, 7) oder auf das Überschwemmungswasser (Wв I: 344,2) angewendet werden. Von daher halte ich es, gerade auch im Hinblick auf die Protasis, die eine dunkel gewordene und dann wieder aufflammende Lampe beschreibt, für das wahrscheinlichste, dass „wiederaufleben“ gemeint ist, und zwar vermutlich in dem Sinne, dass man von einer gefährlichen Krankheit genesen wird oder einer Gefahr entkommt.

Nunmehr kann ich insgesamt zusammenfassen: Die divinatorischen Traktate wurden von der indigenen intellektuellen Elite genutzt und zusammen mit Kompositionen unbezweifelbar wissenschaftlicher Art gelagert. Sie zeigen eine klare und überlegte Textorganisation mit regulierter Ausdrucksweise. Ihre kasuistische Struktur macht sie etwa mit Gesetzestexten vergleichbar. Die allegorische Methode stellt eine Hermeneutik dar, deren Resultate meist gut nachvollziehbar sind, auch wenn sie nicht zwingend sein mögen. Die Ausdrucksweise ist relativ normiert, und in Einzelfällen könnten sogar speziell präzisierte Verwendungen von Wörtern vorliegen. Unter diesen Gegebenheiten plädiere ich dafür, sie als ,wissenschaftlich ${ }^{\text {' }} \mathrm{zu}$ kategorisieren, bzw. festzuhalten, dass der Begriff ,wissenschaftlich', will er für das Alte Ägypten sinnvoll sein, Konnotationen hat, die nicht einfach mit denen unserer postmodernen Welt deckungsgleich sind.

\section{Literaturverzeichnis}

AUFRĖRE, Sydney H. 1985. „Le cœur, l'annuaire gauche, Sekhmet et les maladies cardiaques." Revue d'Égyptologie 36: 21-34.

BEGG, D. J. Ian. 1998. „It was Wonderful, Our Return in the Darkness with ... the Baskets of Papyril Papyrus Finds at Tebtunis from the Bagnani Archives.“ Bulletin of the American Society of Papyrologists 35: 185-210. 
BRESCIANI, Edda. 1981. „Frammenti da un "prontuario legale« demotico da Tebtuni nell'Istituto Papirologico G. Vitelli di Firenze." Egitto e Vicino Oriente 4: 201-215.

BROWN, David. 2000. Mesopotamian Planetary Astronomy-Astrology (Cuneiform Monographs, 18). Groningen: Styx.

ChauveAu, Michel. 1991. „P. Carlsberg 301: Le manuel juridique de Tebtynis“. In: Paul John Frandsen (Hg.), The Carlsberg Papyri 1. Demotic Texts from the Collection (Carsten Niebuhr Institute of Near Eastern Studies Publications, 15). Copenhagen: Museum Tusculanum Press: 103-127.

DONKER VAN HeEL, Koen. 1990. The Legal Manual of Hermopolis [P. Mattha] (Uitgaven vanwege de Stiching "Het Leids Papyrologisch Instituut", 11). Leiden: Papyrologisch Instituut.

ELSBERGEN, Michael Johannes VAN. 1997. Fischerei im alten Ägypten. Untersuchungen zu den Fischfangdarstellungen in den Gräbern der 4. bis 6. Dynastie (Abhandlungen des Deutschen Archäologischen Instituts Kairo, 14). Berlin: Achet.

FINCKE, Jeanette C. 2006-2007. „Omina, die göttlichen ,Gesetze' der Divination. “ Jaarbericht van het Vooraziatisch-Egyptisch Genootschap Ex Oriente Lux 40: 131-147.

FOSSEY, Charles.1921-1922. „Deux principes de la divination assyro-babylonienne d'après le traité Šumma Izbu." Annuaire de l'École pratique des hautes études. Section des sciences religieuses: 1-18.

GAMER-WALLERT, Ingrid. 1970. Fische und Fischkulte im alten Ägypten (Ägyptologische Abhandlungen, 21). Wiesbaden: Harrassowitz.

Gardiner, Alan Henderson. 1935. Hieratic Papyri in the British Museum. Third Series. (Chester Beatty Gift). London: British Museum Press.

HeEßEL, Nils P. 2008. Die Opferschau in Assur. Studien zur Entwicklung und Serialisierung der Opferschau-Kompendien in Babylonien und Assyrien. Habilitationsschrift Heidelberg.

HOFFMANN, Friedhelm. 1995. „Astronomische und astrologische Kleinigkeiten I: Pap. Wien D 6005.“ Enchoria 22: 22-26.

HofFmanN, Friedhelm. 1996. Der Kampf um den Panzer des Inaros. Studien zum P. Krall und seiner Stellung innerbalb des Inaros-Petubastis-Zyklus (Mitteilungen aus der Papyrussammlung der Österreichischen Nationalbibliothek Erzherzog Rainer. Neue Serie, 26). Wien: Hollinek.

Hoffmann, Friedhelm. In Vorbereitung. Der medizinische Papyrus Wien D 6257 und die übrigen ägyptischen medizinischen Texte aus römischer Zeit.

Hornung, Erik. 1967. „Die Bedeutung des Tieres im Alten Ägypten.“ Studium Generale 20: 69-84.

HugHES, George R. 1957. „Rezension Demotisches Glossar W. Erichsen. “ Journal of Near Eastern Studies 16: 55-63.

HuGHES, George R. 1983. „An Astrologer's Handbook in Demotic Egyptian“. In: Leonard H. Lesko (Hg.), Egyptological Studies in Honor of Richard A. Parker Presented at the Occasion of his 78th Birthday December 10, 1983. Hannover/London: University Press of New England: 53-69. 
JANSSEN, Jozef.1946. De traditionelle egyptische Autobiografie vóór het Nieune Rijk. Leiden: Brill.

JASTROW, Morris jr. 1914. Babylonian-Assyrian Birth-Omina and their Cultural Significance (Religionsgeschichtliche Versuche und Vorarbeiten, 14,5). Giessen: Töpelmann.

JEYES, Ulla. 1991-1992. „Divination as a Science in Ancient Mesopotamia.“ Jaarbericht van het Vooraqiatisch-Egyptisch Genootschap Ex Oriente Lux 32: 23-41.

JoHnson, Janet H. 1976. The Demotic Verbal System (Studies in Ancient Oriental Civilisation, 38). Chicago: Oriental Institute.

KAPLONY-HECKEL, Ursula. 1986. Die Giessener hieroglyphischen und demotischen Texte (Kurzberichte aus den Giessener Papyrus-Sammlungen, 42). Giessen: Universitäts-Bibliothek.

KoCH-Westenholz, Ulla. 1995. Mesopotamian Astrology. An Introduction to Babylonian and Assyrian Celestial Divination (Carsten Niebuhr Institute of Near Eastern Studies Publications, 19). Copenhagen: Museum Tusculanum Press.

KRAUS, Fritz R. 1960. „Ein zentrales Problem des altorientalischen Rechtes: Was ist der Codex Hammurabi?" Genava 8: 283-296.

LABAT, René. 1951. Traité akkeadien de diagnostics et prognostics médicaux (Collection de travaux de l'Académie internationale d'histoire des sciences, 7). Leiden: Brill.

LEICHTY, Erle. 1970. The Omen Series Šmma Iz̧bu (Texts from Cuneiform Sources, 4). Locust Valley, N. Y.: Augustin.

LEITZ, Christian. 1994. Tagewählerei: das Buch h3t nhh ph.wy dt und verwandte Texte (Ägyptologische Abhandlungen, 55). Wiesbaden: Harrassowitz.

LEITZ, Christian. 2000. „Altägyptische Traumdeutung nach einem Papyrus des Neuen Reiches“. In: Axel Karenberg und Christian Leitz (Hgg.), Heilkunde und Hochkultur I. Geburt, Seuche und Traumdeutung in den antiken Zivilisationen des Mittelmeerraumes. Münster: LIT Verlag: 221-246.

LIPPERT, Sandra. 2003. „Die sogenannte Zivilprozeßordnung. Weitere Fragmente der ägyptischen Gesetzessammlung." Journal of Juristic Papyrology 33: 91-135.

LIPPERT, Sandra. 2004a. Ein demotisches juristisches Lebrbuch: Untersuchungen zu Papyrus Berlin P 23757 rto (Ägyptologische Abhandlungen, 66). Wiesbaden: Harrassowitz.

LIPPERT, Sandra. 2004b. „Fragmente demotischer juristischer Bücher (pBerlin 23890 a-b, d-g rto und pCarlsberg 628)“. In: Friedhelm Hoffmann und Heinz-Josef Thissen (Hgg.), Res severa verum gaudium. Festschrift für Karl-Theodor Zaurich zum 65. Geburtstag am 8. Juni 2004 (Studia Demotica, 6). Leuven/Paris/ Dudley, MA: Peeters: 389-405.

LIPPERT, Sandra.2008. Einführung in die altägyptische Rechtsgeschichte. Berlin: LIT Verlag.

LIPPERT, Sandra und Maren SCHENTULEIT (Hgg.). 2005. Tebtynis und Soknopaiou Nesos. Leben im römerzeitlichen Fajum. Akten des internationalen Symposions vom 11.-13. Dezember 2003 in Sommerhausen bei Würzburg. Wiesbaden: Harrassowitz. 
LOPRIENO, Antonio. 2000. „Puns and Word Play in Ancient Egyptian“. In: Scott B. Noegel (Hg.), Puns and Pundits. Word Play in the Hebrew Bible and Ancient Near Eastern Literature. Bethesda: Capital Decisions: 3-20.

LORTON, David. 1977. „The Treatment of Criminals in Ancient Egypt. “ Journal of the Economic and Social History of the Orient 20: 2-64.

Mattha, Girgis. 1975. The Demotic Legal Code of Hermopolis West. Preface, Additional Notes and Glossary by George R. Hughes (Bibliothèque d'Étude, 45). Cairo: Institut Français d'Archéologie Orientale.

MAUL, Stefan M. 1994. Zukunftsbewältigung. Eine Untersucbung altorientalischen Denkens anhand der babylonisch-assyrischen Löserituale (Namburbi) (Baghdader Forschungen, 18). Mainz: von Zabern.

Maul, Stefan M. 2003-2005. „Omina und Orakel. A. Mesopotamien“. In: Erich Ebeling, Dietz Otto Edzard, Ernst F. Weidner und Michael P. Streck (Hgg.), Reallexikon der Assyriologie und Vorderasiatischen Archäologie. Bd. 10. Berlin: de Gruyter: 45-88.

MORENZ, Siegfried. 1957. „Rechts und links im Totengericht.“ Zeitschrift für Agyptische Sprache und Altertumskunde 82: 62-71.

MORENZ, Siegfried. 1958/1975. „Wortspiele in Ägypten“. In: Festschrift Johannes Jabn zum 22. November 1957. Leipzig: E. A. Seemann: 23-32. [Wieder abgedruckt in: Elke Blumenthal und Siegfried Herrmann (Hgg.). 1975. Siegfried Morenz, Religion und Geschichte des alten Ägypten. Gesammelte Aufsätze. Weimar: Böhlau: 328-342.]

MORSCHAUSER, Scott N.1991. Threat-Formulae in Ancient Egypt. A Study of the History, Structure and Use of Threads and Curses in Ancient Egypt. Baltimore, Md.: Halgo.

Neugebauer, Otto und Richard Anthony PARKER. 1969. Egyptian Astronomical Texts. Bd. 3: Decans, Planets, Constellations and Zodiacs. Providence, R. I./London: Brown University Press.

NOEgEL, Scott B. 2007. Nocturnal Ciphers: The Allusive Language of Dreams in the Ancient Near East (American Oriental Series, 89). New Haven, Conn.: American Oriental Society.

PARKER, Richard Anthony. 1972. Demotic Mathematical Papyri (Brown Egyptological Studies, 7). Providence, R. I./London: Brown University Press.

Pettinato, Giovanni. 1966. Die Ölwabrsagung bei den Babyloniern (Studi semitici, 21-22). Roma: Istituto di studi del Vicino Oriente.

QUACK, Joachim Friedrich. 1997. „Rezension zu Leitz 1994., Tagewählerei.“ Lingua Aegyptia 5: 277-287.

QUACK, Joachim Friedrich. 2006a. „A Black Cat from the Right, and a Scarab on your Head. New Sources for Ancient Egyptian Divination“. In: Kasia Maria Szpakowska (Hg.), Through a Glass Darkly: Magic, Dreams, and Prophecy in Ancient Egypt. Swansea: Classical Press of Wales: 175-187.

QUACK, Joachim Friedrich. 2006b. „Opfermahl und Feindvernichtung im Altägyptischen Ritual." Mitteilungen der Berliner Gesellschaft für Anthropologie, Ethnologie und Urgeschichte 27: 67-80. 
QUACK, Joachim Friedrich.2006c. „Zu Syntax und Zeitbezug der demotischen zweiten Tempora. "Lingua Aegyptia 14: 251-262.

QUACK, Joachim Friedrich.2008. „Demotische magische und divinatorische Texte“. In: Bernd Janowski und Gernot Wilhelm (Hgg.), Omina, Orakel, Rituale und Beschwörungen (Texte aus der Umwelt des Alten Testaments. Neue Folge, 4). Gütersloh: Gütersloher Verlagshaus: 331-385.

QUACK, Joachim Friedrich. 2009. Einführung in die altägyptische Literaturgeschichte III. Die demotische und gräko-ägyptische Literatur (Einführungen und Quellentexte zur Ägyptologie, 3). 2. Aufl. Berlin: LIT Verlag.

QUACK, Joachim Friedrich. 2010. „Aus zwei spätzeitlichen Traumbüchern (Pap. Berlin P. 29009 und 23058)“. In: Hermann Knuf, Christian Leitz und Daniel von Recklinghausen (Hgg.), Honi soit qui mal y pense. Studien zum pharaonischen, griechisch-römischen und spätantiken Ägypten zu Ebren von Heinz-Josef Thissen (Orientalia Lovaniensia Analecta, 194). Leuven/Paris/Walpole, MA: Peeters: 99-110.

QUACK, Joachim Friedrich. Im Druck. „Fragmente eines änigmatischen Weisheitstextes (Ex pOxy. 79/103). Mit Bemerkungen zu den pythagoräischen Akousmata und der spätägyptischen Weisheitstradition“. In: Didier Devauchelle und Ghislaine Widmer (Hgg.), Akten des Demotistenkongresses. Paris.

QUACK, Joachim Friedrich. In Vorbereitung. „Schicksalsvorstellungen im späten Ägypten“.

QUACK, Joachim Friedrich und Kim RYHOLT. In Vorbereitung. „Manuals of Dream Interpretation“. In: The Carlsberg Papyri 11.

RiTTER, Jim. 2010. „Translating Rational-Practice Texts“. In diesem Band: 339373.

RoCHBERG, Francesca. 1999. „Empiricism in Babylonian Omen Texts and the Classification of Mesopotamian Divination as Science." Journal of the American Oriental Society 119: 559-569.

RyHolt, Kim. 1999. The Carlsberg Papyri 4. The Story of Petese, Son of Petetum and Seventy Other Good and Bad Stories (Carsten Niebuhr Institute of Near Eastern Studies Publications, 23). Copenhagen: Museum Tusculanum Press.

RYHOLT, Kim. 2005. „On the Contents and Nature of the Tebtunis Temple Library. A Status Report“. In: Sandra Lippert und Maren Schentuleit (Hgg.), Tebtynis und Sokenopaiou Nesos. Leben im römerzeitlichen Fajum. Akten des internationalen Symposions vom 11.-13. Dezember 2003 in Sommerhausen bei Würzburg. Wiesbaden: Harrassowitz: 141-170.

SANDER-HANSEN, Constantin Emil. 1948. „Die phonetischen Wortspiele des ältesten Ägyptisch.“ Acta Orientalia 20: 1-22.

SEIDL, Erwin. 1963. „Das Giessener Fragment zu dem Bruchstück einer demotischen Zivilprozeßordnung. " Kurzbericbte aus den Giessener Papyrus-Sammlungen 16: 3-10.

SETHE, Kurt und Josef PARTSCH. 1920. Demotische Urkunden zum altägyptischen Bürgschaftsrechte vorzüglich der Ptolemäerzeit (Abhandlungen der Sächsischen Akademie der Wissenschaften zu Leipzig. Phil.-hist. Kl., 32). Leipzig: Teubner. 
SETHE, Kurt und Wilhelm SPIEGELBERG. 1929. Zwei Beiträge zu dem Bruchstück einer ägyptischen Zivilprozeßordnung in demotischer Schrift (Abhandlungen der Bayerischen Akademie der Wissenschaften. Neue Folge, 4). München: Bayerische Akademie der Wissenschaften.

SIMPSON, RobertS. 1996. Demotic Grammar in the Ptolemaic Sacerdotal Decrees. Oxford: Griffith Institute.

SPIEGElBERG, Wilhelm. 1902. Demotische Papyrus aus den königlichen Museen zu Berlin. Leipzig/Berlin: Giesecke \& Devrient.

SPIEGELBERG, Wilhelm.1925. Demotica I (Sitzungsberichte der Bayerischen Akademie der Wissenschaften, 6). München: Bayerische Akademie der Wissenschaften.

SPIEGELBERG, Wilhelm. 1929. Aus einer ägyptischen Zivilprozeßordnung der Ptolemäerzeit (Abhandlungen der Bayrischen Akademie der Wissenschaften. Neue Folge, 1). München: Bayerische Akademie der Wissenschaften.

Sснотт, Siegfried und Erika Sснотт. 1990. Bücher und Bibliotheken im Alten Agypten. Verzeichnis der Buch- und Spruchtitel und der Temini technici. Wiesbaden: Harrassowitz.

SOMMERFELD, Walter. 2000. „Traumdeutung als Wissenschaft und Therapie im Alten Orient". In: Axel Karenberg und Christian Leitz (Hgg.), Heilkeunde und Hochkultur I. Geburt, Seuche und Traumdeutung in den antiken Zivilisationen des Mittelmeerraumes. Münster: LIT Verlag: 201-219.

STARR, Ivan. 1983. The Rituals of the Diviner (Bibliotheca Mesopotamica, 12). Malibu: Undena.

SzPakowskA, Kasia M. 2003. Behind Closed Eyes. Dreams and Nightmares in Ancient Egypt. Swansea: Classical Press of Wales.

TAIT, William J.1977. Papyri from Tebtunis in Egyptian and Greek (Texts from Excavations, 3). London: Egypt Exploration Society.

TAIT, William J.1991a. „P. Carlsberg 230: Eleven Fragments from a Demotic Herbal“. In: Paul John Frandsen (Hg.), The Carlsberg Papyri 1. Demotic Texts from the Collection (Carsten Niebuhr Institute of Near Eastern Studies Publications, 15). Copenhagen: Museum Tusculanum Press: 47-92.

TAIT, William J. 1991b. „P. Carlsberg 236: Another Fragment of a Demotic Legal Manual“. In: Paul John Frandsen (Hg.), The Carlsberg Papyri 1. Demotic Texts from the Collection (Carsten Niebuhr Institute of Near Eastern Studies Publications, 15). Copenhagen: Museum Tusculanum Press: 93-99.

TILL, Walter C. 1966. Koptische Grammatik (Saïdischer Dialekt). Leipzig: VEB Verlag Enzyklopädie.

VAlbelle, Dominique. 1985. ,Les ouvriers de la tombe: Deir el-Médineb à l'époque ramesside (Bibliothèque d'Étude, 96). Cairo: Institut Français d'Archéologie Orientale.

VERnUS, Pascal. 1981. „Omina calendériques et compatibilité d'offrandes sur une tablette hiératique de la XVIIIe dynastie.“ Revue d'Égyptologie 33: 90-124.

VolTEN, Aksel. 1942. Demotische Traumdeutung (Pap. Carlsberg XIII und XIV Verso), (Analecta Aegyptiaca, 3). Copenhagen: Munksgaard. 
WestbrooK, Raymond. 1985. „Biblical and Cuneiform Law Codes.“ Revue Biblique 92: 247-264.

YARON, Reuven. 1988. The Laws of Eshnunna. Jerusalem/London: Brill.

ZAUZICH, Karl-Theodor. 1980. „Aus zwei demotischen Traumbüchern. “ Archiv für Papyrusforschung und verwandte Gebiete 27: 91-98.

ZAUZICH, Karl-Theodor. 1991. „Einleitung“. In: Paul John Frandsen (Hg.), The Carlsberg Papyri 1. Demotic Texts from the Collection (Carsten Niebuhr Institute of Near Eastern Studies Publications, 15). Copenhagen: Museum Tusculanum Press: $1-11$.

ZgolL, Annette. 2006. Traum und Weiterleben im antiken Mesopotamien (Alter Orient und Altes Testament, 333). Münster: Ugarit-Verlag. 